\title{
A Note on the Transformability of Spherically Symmetric Metrics
}

\author{
By Paul Kustaanheimo
}

(Communicated by G. C. McVittie.)

(Received 30th December, 1949. Read 3rd March, 1950.)

Summary. It is shown that every spherically symmetric metric can be transformed into the isotropic form. As illustration an example is given.

Max Wyman has in Mathematical Reviews, 10 (1949), 579, reviewed a paper by Bertil Qvist and the author ${ }^{1}$ and in the review declared that:

"It should be noted that the authors state that every spherically symmetric metric can be written in the so-called isotropic form. This assertion is incorrect as it is based on a proof given by Tolman [Relativity, Thermodynamics and Cosmology (Oxford, 1934), p. 240] which is wrong. The right-hand side of formula (94.7) as given by Tolman is not a perfect differential when $\lambda$ is a function of $t . "$

In view of the above remark I venture to put forward a few observations.

Einstein and Straus have stated" that "A general centrally-symmetric field can be brought into the (conformally Euclidean, not necessarily static) form

$$
d s^{2}=-e^{\mu} \delta_{i k} d x_{i} d x_{k}+e^{\nu} d t^{2} \quad i, k=1,2,3,
$$

where $\mu$ and $\nu$ are functions of $r$ and $t "$.

This transformation of a general spherically symmetric metric into the isotropic form can be performed in the following way.

As is known, every spherically symmetric metric may be written in the standard form

$$
d s^{2}=e^{\nu} d t^{2}-r^{2}\left(d \theta^{2}+\sin ^{2} \theta d \phi^{2}\right)-e^{\lambda} d r^{2},
$$

where $\nu$ and $\lambda$ are certain functions of $r$ and $t$ alone.

Paul Kustaanheimo and Bertil Qvist, "A note on some general solutions of the Einstein field equations in a spherically symmetric world ", Soc. Sc. Fenn. Comm. Phys.Math. XIII, No. 16 (1948).

2 Albert Einstein and Ernst G. Straus, "The Influence of the Expansion of Space on the Gravitation Fields Surrounding the Individual Stars ", Reviews of Modern Physics, Vol. 17, Nos. 2 and 3 (1945), 121. 
We make in (2) the differential substitution

$$
\begin{aligned}
& d \bar{r}=A d r+B d t \\
& d \bar{t}=C d r+D d t
\end{aligned}
$$

where $A, B, C$ and $D$ are for the present arbitrary functions of $r$ and $t$, which have to satisfy only the integrability conditions

$$
\begin{aligned}
& \frac{\partial A}{\partial t}=\frac{\partial B}{\partial r}, \\
& \frac{\partial C}{\partial t}=\frac{\partial D}{\partial r} .
\end{aligned}
$$

Since by (3) and (4)

$$
d r=\frac{D d \bar{r}-B d \bar{t}}{A \bar{D}-B \bar{C}}, \quad d t=\frac{-C d \bar{r}+A d \bar{t}}{A D-B C},
$$

we have from (2)

$$
\begin{aligned}
d s^{2}=\frac{e^{\nu} A^{2}-e^{\lambda} B^{2}}{(A D-B C)^{2}} d \bar{t}^{2}-r^{2}\left(d \theta^{2}\right. & \left.+\sin ^{2} \theta d \phi^{2}\right) \\
& -\frac{e^{\lambda} D^{2}-e^{\nu} C^{2}}{(A D-B C)^{2}} d \bar{r}^{2}-2 \frac{e^{\nu} A C-e^{\lambda} B D}{(A D-B C)^{2}} d \bar{r} d \bar{t} .
\end{aligned}
$$

The expression (7) is of the form (1) if

$$
\begin{aligned}
e^{\nu} A C-e^{\lambda} B D & =0, \\
\frac{1}{\bar{r}} A D-\frac{1}{\bar{r}} B C & =\frac{1}{r} \sqrt{e^{\lambda} D^{2}-e^{\nu} C^{2}},
\end{aligned}
$$

or

$$
\begin{aligned}
& \frac{A}{\bar{r}}=\frac{r^{-1} e^{\lambda} D}{\sqrt{e^{\lambda} D^{2}-e^{r} C^{2}}}, \\
& \frac{B}{\bar{r}}=\frac{r^{-1} e^{\nu} C}{\sqrt{e^{\lambda} D^{2}-e^{\nu} C^{2}}} .
\end{aligned}
$$

Since (3) is a total differential only when also

$$
\frac{d \bar{r}}{\bar{r}}=\frac{A}{\bar{r}} d r+\frac{B}{\bar{r}} d t
$$

is a total differential, we may use instead of (5) the equivalent condition

$$
\frac{\partial}{\partial t} \frac{A}{\bar{r}}=\frac{\partial}{\partial r} \frac{B}{\bar{r}}
$$

Then we get, according to (8) and (9),

$$
\frac{\partial}{\partial t} \frac{r^{-1} e^{\lambda} D}{\sqrt{e^{\lambda} D^{2}-e^{\nu} C^{2}}}=\frac{\partial}{\partial r} \frac{r^{-1} e^{\nu} C}{\sqrt{e^{\lambda} D^{2}-e^{\nu} C^{2}}}
$$


The equation (10) really contains only one unknown function, the ratio $C / D$. Putting $R=C / D$ and carrying out the differentiations in (10), we get

$$
\begin{aligned}
e^{\lambda+\nu} R \frac{\partial R}{\partial t}- & e^{\lambda}+\nu \frac{\partial R}{\partial r}+\left(\frac{1}{2} e^{\nu} \frac{\partial}{\partial r} e^{\nu}-\frac{1}{r} e^{2 \nu}\right) R^{3} \\
& +\left(\frac{1}{2} e^{\lambda} \frac{\partial}{\partial t} e^{\nu}-e^{\nu} \frac{\partial}{\partial t} e^{\lambda}\right) R^{2}+\left(\frac{1}{2} e^{\nu} \frac{\partial}{\partial r} e^{\lambda}-e^{\lambda} \frac{\partial}{\partial r} e^{\nu}+\frac{1}{r} e^{\lambda+\nu}\right) R \\
& +\frac{1}{2} e^{\lambda} \frac{\partial}{\partial t} e^{\lambda}=0
\end{aligned}
$$

which is of the general form

$$
P(r, t, R) \frac{\partial R}{\partial t}+Q(r, t) \frac{\partial R}{\partial r}-S(r, t, R)=0
$$

and has an infinite number of solutions in all cases considered in the theory of relativity.

After taking any one of the solutions of (11) as the ratio $R=C / D$, it is always possible to find a function $D$ which satisfies (6), an equation that may be written

$$
\frac{1}{R} \frac{\partial}{\partial r} \ln D-\frac{\partial}{\partial t} \ln D=\frac{\partial}{\partial t} \ln R
$$

where $R=C / D$ is a known function of $r$ and $t$. Finally, from (8) and (9) we get $A / \bar{r}$ and $B / \bar{r}$, which, together with $C$ and $D$, when substituted into (3) and (4), give the transformation

$$
\frac{d \bar{r}}{\bar{r}}=\frac{A}{\bar{r}} d r+\frac{B}{\bar{r}} d t, \quad d \bar{t}=C d r+D d t .
$$

By means of this transformation, (2) changes into the isotropic form

$$
d s^{2}=\frac{e^{\lambda+\nu}}{e^{\lambda} D^{2}-e^{\nu} C^{2}} d \bar{t}^{2}-\frac{r^{2}}{\bar{r}^{2}}\left[d \bar{r}^{2}+\bar{r}^{2}\left(d \theta^{2}+\sin ^{2} \theta d \phi^{2}\right)\right]
$$

If in particular the metric (2) is static or quasistatic, i.e. if $\lambda$ is a function of $r$ alone, we may immediately take $C / D=0$ as a solution of (10), and then $D=1$ as a solution of (6).

The transformation (3)... (4) now takes the form

$$
\frac{d \bar{r}}{\bar{r}}=e^{i \lambda} \frac{d r}{r}, \quad d \bar{t}=d t,
$$

which is given in Tolman, op. cit., p. 240, formula (94.7).

As an application we transform the metric

into isotropic form.

$$
d s^{2}=\frac{r^{2}}{t^{2}} d t^{2}-r^{2}\left(d \theta^{2}+\sin ^{2} \theta d \phi^{2}\right)-\frac{r^{2}}{t} d r^{2}
$$


The metric (13) is representable in the standard form (2), by choosing

$$
\begin{aligned}
& \lambda=2 \ln r-\ln t, \\
& \nu=2 \ln r-2 \ln t .
\end{aligned}
$$

The equation (11) thus takes the form

$$
R \frac{\partial R}{\partial t}-\frac{\partial R}{\partial r}-\frac{1}{2}=0
$$

the general solution of which is

$$
2 R+r=F\left(R^{2}-t\right),
$$

where $F($ ) is an arbitrary function of one variable. We may in particular choose $F()$ to be identically zero. Then we have

$$
R=\frac{C}{D}=-\frac{r}{2}
$$

and so (6) takes the form

$$
-\frac{r}{2} \frac{\partial}{\partial t} D=\frac{\partial}{\partial r} D
$$

one solution being

$$
D=4 t-r^{2} .
$$

The transformation (3)... (4) thus has the form

or

$$
\begin{gathered}
\frac{d \bar{r}}{\bar{r}}=\frac{2 d r-r t^{-1} d t}{\sqrt{4 t-r^{2}}}, \\
d \bar{t}=\left(4 t-r^{2}\right)\left(-\frac{1}{2} r d r+d t\right), \\
\bar{r}=e^{2 \arcsin \frac{1}{2} r / v t} \\
\bar{t}=\frac{1}{8}\left(4 t-r^{2}\right)^{2},
\end{gathered}
$$

transforming the metric (13) into the isotropic form

$$
d s^{2}=\frac{4 r^{2}}{t\left(4 t-r^{2}\right)^{3}} d \bar{t}^{2}-\frac{r^{2}}{\bar{r}^{2}}\left[d \bar{r}^{2}+\bar{r}^{2}\left(d \theta^{2}+\sin ^{2} \theta d \phi^{2}\right)\right]
$$

or $\quad d s^{2}=\frac{\sin ^{2} \frac{1}{2} \ln \bar{r}}{\bar{t} \sqrt{2 \bar{t}}} d \bar{t}^{2}-\frac{2}{\bar{r}^{2}} \sqrt{2 \bar{t}} \tan ^{2} \frac{1}{2} \ln \bar{r}\left[d \bar{r}^{2}+\bar{r}^{2}\left(d \theta^{2}+\sin ^{2} \theta d \phi^{2}\right)\right]$.

Astronomical Observationy,

Helsinki, Finland. 